\title{
Improvement of Depression in a Patient with Hypothyroidism and Deiodinase Polymorphism with LT3 Therapy
}

\author{
Ziyan Ahmed ${ }^{1}$, Rinsha Sherin ${ }^{1}$, Thanh Hoang ${ }^{1}$, Tatiana Fonseca ${ }^{2}$, and Mohamed Shakir ${ }^{1}$ \\ ${ }^{1}$ Walter Reed National Military Medical Center \\ ${ }^{2}$ University of Chicago Andy Minn Oncology Lab
}

September 25, 2021

\begin{abstract}
We report a 54-year-old man with treatment-resistant depression (TRD) and hypothyroidism who responded to LT3/LT4 combination, rather than LT4 alone. He was able to discontinue all antidepressant medications eventually. Interestingly, the patient has a DIO2 polymorphism.
\end{abstract}

Improvement of Depression in a Patient with Hypothyroidism and Deiodinase Polymorphism with LT3 Therapy

Ziyan S. Ahmed ${ }^{1}$, Rinsha PV Sherin ${ }^{1}$, Tatiana L. Fonseca ${ }^{2}$, Thanh D. Hoang ${ }^{1,3}$, and Mohamed KM. Shakir ${ }^{1,3}$

${ }^{1}$ Endocrinology Division, Walter Reed National Military Medical Center, Bethesda, MD

${ }^{2}$ Section of Adult and Pediatric Endocrinology, Diabetes \& Metabolism,

University of Chicago, Chicago, IL

${ }^{3}$ Endocrinology Division, Uniformed Services University of the Health Sciences, Bethesda, MD

Short title : Improvement of Depression with LT3/LT4

Keywords: depression, T3, hypothyroidism, polymorphism

Corresponding Author : Dr. Thanh D. Hoang

Division of Endocrinology

Walter Reed National Military Medical Center,

8901 Wisconsin Ave,

Bethesda, MD 20889.

E-mail: tdhdthanh@gmail.com

Disclaimer: The views expressed in this article are those of the authors and do not reflect the official policy of the Department of Army/Navy/Air Force, Department of Defense, or the U.S. Government.

Disclosure Statement : The authors have nothing to disclose. The patient was originally enrolled in study protocol Clinical Trials \#NCT02317926.

Funding statement : None

ABSTRACT 
We report a 54-year-old man with treatment-resistant depression (TRD) and hypothyroidism who responded to LT3/LT4 combination, rather than LT4 alone. He was able to discontinue all antidepressant medications eventually. Interestingly, the patient has a DIO2 polymorphism.

\section{Key Clinical Message}

There is a good correlation between the depression scores and the serum T3 levels. In hypothyroid patients with functional DIO2 polymorphism, thrice-daily LT3 dosing may significantly improve depression. Additional studies are needed.

\section{INTRODUCTION}

Previous studies have shown a strong association between hypothyroidism and depressive disorders (1-3). Treatment-resistant depression (TRD) is traditionally defined as depression with inadequate clinical response usually following the traditional antidepressant therapy $(4,5)$. Patients with depression who fail to achieve symptom remission may pose significant clinical challenges and need augmentation pharmacology approach $(4,5)$. We report a patient with TRD, hypothyroidism, and Thr92 Ala-DIO 2 polymorphism who has responded well to T3 therapy, given in 3 divided doses.

\section{METHODS}

Because of the ongoing TRD he was followed only with General health questionnaire (GHQ), Thyroid symptom questionnaire (TSQ), Beck Depression inventory scores (BDI-II) (6-8) and measurements of thyroid functions. Genotyping was performed per Allelic Discrimination protocol from real-time PCR machine (Applied Biosciences) using Taqman reagents and rs225014 SNP primer $(9,10)$. Statistical analysis was performed by analysis of variance (ANOVA) and post-hoc analysis by Tukey test.

\section{CASE REPORT}

A 54-year-old man presented to the emergency room with suicidal ideation 5 years ago. Additionally, patient had severe depression and an immense desire to surf the internet, generally prohibited sites. He also noted a 12-pound weight gain, dry skin, and cold intolerance. Family history was significant for Hashimoto's thyroiditis in one daughter. Physical examination showed HR $64 \mathrm{bpm}$, BP 140/90 mm Hg. Thyroid was diffusely enlarged, approximately 40 grams without palpable nodules. Rest of the examination was normal, except for delayed deep tendon reflexes. Laboratory values at admission: normal CBC, CMP, and serum B12 level. Thyroid functions: serum TSH $560 \mu \mathrm{IU} / \mathrm{mL}$ (normal 0.41-4.2), FT4 $0.20 \mathrm{ng} / \mathrm{dL}$ (normal 0.902.18), total T3 $38 \mathrm{ng} / \mathrm{dL}$ (normal 59-174), TPO antibody $278 \mathrm{IU} / \mathrm{mL}$ (normal 0-34), TG antibody $9.8 \mathrm{ng} / \mathrm{dL}$ (normal 0.0-0.9). Ultrasound confirmed a heterogeneously enlarged thyroid consistent with Hashimoto's thyroiditis. Diagnoses of major depressive disorder and primary hypothyroidism were made. GHQ, TSQ and BDI-II scores obtained 2 days after admission were markedly abnormal (during the 8 week basal period, the symptom scores and thyroid functions were measured at 2 to 3 week intervals $(n=4)$ and these functions were GHQ, $26.0 \pm 8.49$, TSQ $28.5 \pm 7.77$, BDI-II $45.0 \pm 6.18$, TSH $165 \pm 133 \mu \mathrm{IU} / \mathrm{mL}$, Free T4 $0.79 \pm$ $0.25 \mathrm{ng} / \mathrm{dL}$, and total T3 $60.5 \pm 7.89 \mathrm{ng} / \mathrm{dL}$ ) . Patient was treated with levothyroxine (LT4) 175mcg daily and citalopram $20 \mathrm{mg}$ daily. The dose of citalopram was gradually increased to $40 \mathrm{mg}$ daily and amitriptyline $50 \mathrm{mg}$ at bedtime was added along with twice weekly psychotherapy sessions. After 8 weeks of treatment there was modest improvement in GHQ, TSQ, and BDI-II despite normal serum TSH $1.34 \mu \mathrm{IU} / \mathrm{mL}$ (Table 1, Figures 1,2). Three months later, because of the poor response to antidepressant drugs aripiprazole and buspirone were added. After 9 months, LT3 5 mcg once daily was added. Patient continued LT4 + LT3 
5mcg combination for another 13 months and at this time the treatment was changed to LT4 only. Despite being on 4 antidepressant drugs and LT4, his depression scores and internet addiction did not improve significantly (Table 1). Approximately $2 \frac{1}{2}$ years after the initial visit, patient was placed on LT3 $5 \mathrm{mcg}$ thrice daily with reduced dose of LT4. Within 4 weeks of starting LT4 + LT3 TID dosing, patient noted remarkable improvement in depression, suicidal ideation, and internet addiction. It was possible to gradually discontinue all the antidepressant drugs over the next 9 months (Table 1). However, the psychiatrist prescribed over the counter antidepressant drugs, S-adenosylmethionine (SAMe), (Pure Encapsulations ${ }^{\mathrm{TM}}$, Sudbury MA) 1600 $\mathrm{mg} /$ day, and Rhodiola rosea (rosavins $>3.0 \%$, salidroside $>0.8 \%$ at a ratio of $3: 1$, Pure Encapsulations ${ }^{\mathrm{TM}}$, Sudbury MA) $200 \mathrm{mg}$ twice daily. The serum total T3 levels remained in the upper normal range while the patient was taking LT4 +LT3 5mcg TID dosing and the serum TSH levels remained in the lower normal range. A follow up cardiac evaluation was completely normal. He presently continues psychotherapy visits only every 3 months. Additionally, he is gainfully employed and also continues a happy married life. A genetic screen performed for DIO2 polymorphism confirmed that the patient carries one allele of Thr92 Ala-DIO 2 polymorphism. The GHQ, TSQ and BDI-II scores correlated well with serum TSH and T3 levels (Figures 1,2), but less significant with serum free T4 levels (correlation coefficient (r) for GHQ: 0.52, $\mathrm{r}$ for TSQ: 0.62, $\mathrm{r}$ for BDI-II 0.66, Figure 3)

\section{DISCUSSION}

While there is no universally agreed upon definition of TRD (4), it is well accepted that patients with TRD have a poor prognosis. Although our patient was treated with four antidepressant drugs, there was inadequate response in depression and internet addiction. With a diagnosis of primary hypothyroidism, patient was treated with a weight-based dose of levothyroxine as recommended by the American Thyroid Association (11) and the patient achieved euthyroid status within 6 weeks. However, his serum T3 levels were in the low normal range. In a significant proportion of patients with hypothyroidism treated with levothyroxine, serum T3 levels may remain in the low normal range and the exact significance of this is not known (12). Finally, when he received LT3 in 3 divided doses, there was a marked improvement in his depressive symptoms, internet addiction and GHQ, TSQ and BDI-II scores. However, it was noted that his serum TSH levels were in low normal range. Patient had no signs/symptoms of hyperthyroidism and a normal cardiac screening. He was able to discontinue all prescribed antidepressant drugs, and was treated with 2 over- the-counter drugs namely S-adenosylmethionine (SAMe) (13) and Rhodiola Rosea supplements (14). Additionally, our patient required psychotherapy sessions less frequently. Thus, the LT4 + LT3 TID, SAMe, and Rhodiola Rosea combination therapy resulted in significant improvement in his major depressive disorder and the associated internet addiction, an obsessive neurosis and he is able to lead a normal life. There have been considerable controversies regarding what constitutes TRD. However, the inadequate response to trials of 4 antidepressant drugs met to criteria of TRD in our patient. In contrast to the well-accepted treatment of hypothyroidism with LT4, LT3 is often prescribed in depression as an augmentation pharmacotherapy (15). Limited studies utilizing LT3 therapy were conducted as an augmentation of tricyclic antidepressant and found improvement in depression $(15,16)$. Open label studies have also shown some benefits (16). However, there was no superior benefit compared to lithium, although the side effects were much less (16).

Thyroid hormone may play a significant role in noradrenergic and serotonergic neurotransmission as well as in the pathogenesis of depression. However, screening patients with depression for hypothyroidism is not widely supported $(17)$. Previous studies $(18,19)$ have shown an association between immune thyroid diseases and depression, although in one study of subjects with normal thyroid function there was no association between TPO antibodies and depression (20). Our patient had markedly elevated thyroid antibodies and it is not clear whether there was any association between the antibodies and depression.

The use of LT4/LT3 combination therapy or LT3 alone treatment in depression remains controversial. Panicker et al (21) showed that patients with a functional polymorphism in $\mathrm{DIO}_{2}$ may respond better with a LT4/LT3 combination treatment. Compared to previous studies in which T3 supplementation has not been 
beneficial, our patient differs in several ways. Our patient had complete failure of thyroid gland as evidenced by low T4 levels and the need for full body-weight adjusted replacement dose of LT4. It is also unlikely that the improvement resulted from a placebo effect for several reasons. Our patient had already taken T4 alone on 2 occasions and LT4+LT3 once daily previously without improvement. In addition to subjective sense of wellness, the GHQ, TSQ and BDI II scores previously documented as measures of improvements in hypothyroid patients from our institution $(6,7)$ also confirming the benefits on several occasions. Finally, our patient had well documented TRD. However, re-challenging the patient with a higher dose LT4 alone, to keep the TSH in the low normal range would have confirmed but we felt it is unethical. Additionally, since our patient noted significant improvements, he would have refused any modification of the current treatment utilizing LT4+LT3 combination. Since there is only $\mathrm{D}_{2}$ in the brain which converts LT4 to LT3, it is possible that when hypothyroid patients with Thr92 Ala-DIO 2 polymorphism is treated with a thrice daily LT3 regimen, they may respond much better as evidenced by improvements in TRD and various scores. In patients with carriers of the Thr92Ala-DIO ${ }_{2}$ polymorphism, there may be subtle changes in thyroid hormone homeostasis and accumulation of Ala92- $\mathrm{D}_{2}$ in the trans-Golgi apparatus providing less T3 locally (22). These patients may be at higher risk for brain degenerative disease even if they maintain euthyroid. It is possible that the steady state of serum T3 levels, in the high normal range may have been able to at least partially overcome the monocarboxylate transporter (MCT) system especially MCT8 as suggested by Jo et al (23).

In conclusion in our hypothyroid patient with TRD and Thr92Ala-DIO ${ }_{2}$ polymorphism, thrice daily administration of low doses of LT3 improved his depression significantly. Further studies are needed.

\section{Statement of author contribution:}

ZSA - author

RPVS - reviewer

TLF- reviewer, genetic researcher

TDH- reviewer

MKMS- reviewer

Conflict of interest: None to declare.

Ethical approval statement : The manuscript has been reviewed and approved by the IRB and Public Affairs Office.

Consent statement: the authors have confirmed that patient consent has been signed and collected in accordance with the journal's patient consent policy.

\section{References}

1.Ittermann T, Völzke H, Baumeister SE, Appel K, Grabe HJ. Diagnosed thyroid disorders are associated with depression and anxiety. Soc Psychiatry Psychiatr Epidemiol. 2015;50(9):1417-25.

2. Demartini B, Masu A, Scarone S, Pontiroli AE, Gambini O. Prevalence of depression in patients affected by subclinical hypothyroidism. Panminerva Med. 2010;52(4):277-82.

3 Chueire VB, Romaldini JH, Ward LS. Subclinical hypothyroidism increases the risk for depression in the elderly. Arch Gerontol Geriatr. 2007;44(1):21-8

4. Ruberto VL, Jha MK, Murrough JW. Pharmacological Treatments for Patients with Treatment-Resistant Depression. Pharmaceuticals (Basel). 2020;13(6):E116. 
5. Lam RW, Hossie H, Solomons K, Yatham LN. Citalopram and bupropion-SR: combining versus switching in patients with treatment-resistant depression. J Clin Psychiatry. 2004;65(3):337-40.

6. Clyde PW, Harari AE, Getka EJ, Shakir KMM. Combined Levothyroxine Plus Liothyronine Compared With Levothyroxine Alone in Primary Hypothyroidism: A Randomized Controlled Trial. JAMA. 2003;290(22):2952-2958.

7. Hoang TD, Olsen CH, Mai VQ, Clyde PW, Shakir MK. Desiccated thyroid extract compared with levothyroxine in the treatment of hypothyroidism: a randomized, double-blind, crossover study. J Clin Endocrinol Metab. 2013 May;98(5):1982-90.

8. Beck AT, Steer RA, Ball R, Ranieri W. Comparison of Beck Depression Inventories -IA and -II in psychiatric outpatients. J Pers Assess. 1996;67(3):588-97.9

9. Peeters RP, van Toor H, Klootwijk W, et al. Polymorphisms in thyroid hormone pathway genes are associated with plasma TSH and iodothyronine levels in healthy subjects. J Clin Endocrinol Metab. 2003;88(6):28808 .

10. Mentuccia D, Proietti-Pannunzi L, Tanner K, et al. Association between a novel variant of the human type 2 deiodinase gene Thr92Ala and insulin resistance: evidence of interaction with the Trp64Arg variant of the beta-3-adrenergic receptor. Diabetes. 2002;51(3):880-3.

11 Jonklaas J, Bianco AC, Bauer AJ, et al. Guidelines for the treatment of hypothyroidism: prepared by the american thyroid association task force on thyroid hormone replacement. Thyroid. 2014;24(12):1670-751.

12 Gullo D, Latina A, Frasca F, Le Moli R, Pellegriti G, Vigneri R. Levothyroxine monotherapy cannot guarantee euthyroidism in all athyreotic patients. PLoS One. 2011;6(8):e22552.

13 Cuomo A, Beccarini Crescenzi B, Bolognesi S, et al. S-Adenosylmethionine (SAMe) in major depressive disorder (MDD): a clinician-oriented systematic review. Ann Gen Psychiatry. 2020;19:50.

14 Konstantinos, Fanaras \& Heun, Reinhard. (2020). The effects of Rhodiola Rosea supplementation on depression, anxiety and mood - A Systematic Review. Global Psychiatry. 3. 10.2478/gp-2019-0022.

15 .Kelly TF, Lieberman DZ. Long term augmentation with T3 in refractory major depression. J Affect Disord. 2009;115(1-2):230-3.

16. Nierenberg AA, Fava M, Trivedi MH, et al. A comparison of lithium and T(3) augmentation following two failed medication treatments for depression: a STAR*D report. Am J Psychiatry. 2006;163(9):1519-30.

17. Kim JS, Zhang Y, Chang Y, et al. Subclinical Hypothyroidism and Incident Depression in Young and Middle-Age Adults. J Clin Endocrinol Metab. 2018;103(5):1827-33.

18. Pop VJ, Maartens LH, Leusink G, et al. Are autoimmune thyroid dysfunction and depression related. J Clin Endocrinol Metab. 1998;83(9):3194-7.19. Kirim S, Keskek SO, Köksal F, 19. Haydardedeoglu FE, Bozkirli E, Toledano Y. Depression in patients with euthyroid chronic autoimmune thyroiditis. Endocr J. 2012;59(8):705-8.

20. Engum A, Bjøro T, Mykletun A, Dahl AA. Thyroid autoimmunity, depression and anxiety; are there any connections? An epidemiological study of a large population. J Psychosom Res. 2005;59(5):263-8.

21. Panicker V, Saravanan P, Vaidya B, et al. Common variation in the DIO2 gene predicts baseline psychological well-being and response to combination thyroxine plus triiodothyronine therapy in hypothyroid patients. J Clin Endocrinol Metab. 2009;94(5):1623-9.

22. McAninch EA, Rajan KB, Evans DA, et al. A Common DIO2 Polymorphism and Alzheimer Disease Dementia in African and European Americans. J Clin Endocrinol Metab. 2018;103(5):1818-26.23.

23.Jo S, Fonseca TL, Bocco BMLC, et al. Type 2 deiodinase polymorphism causes ER stress and hypothyroidism in the brain. J Clin Invest. 2019;129(1):230-45. 


\section{Abbreviations:}

BDI-II: Beck Depression Inventory

TSH: Thyroid Stimulating Hormone

Free T4: Free Thyroxine

Total T3: Total Triiodothyronine

\section{Legends to Figures}

Figure 1: Showing the relationship between serum TSH (thyroid stimulating hormone) and GHQ, TSQ and BDI-II.

Figure 2: Showing the relationship between serum total T3 (triiodothyronine) levels and GHQ, TSQ, and BDI-II.

Figure 3: Showing the relationship between free T4 (thyroxine) levels and GHQ, TSQ, and BDI-II.

\section{Notes for the table}

Beck Depression Inventory-II (BDI-II), consisting of 21 items assessing specific cognitive, affective, and physical symptoms of depression, corresponds well to a clinical diagnosis of depressive disorders outlined in the Diagnostic and Statistical Manual of Mental Disorders, Fourth Edition (8-10). The total BDI-II is the sum of all item scores. The minimum score is 0 , and maximum score is 63 . The BDI-II scores of 0-13 denote no/minimal depression, scores of 14-19 denote mild depression, scores of 20-28 denote moderate depression, and scores of 29-63 denote severe depression.

Validity of these tests in patients with hypothyroidism has been well established previously (8.9). Serum TSH, free T4, and total T3 were measured by the electrochemiluminescence immunoassay (ECLIA, Cobas 8000, K Diagnostics, Indianapolis, IN). DNA was extracted from blood samples.
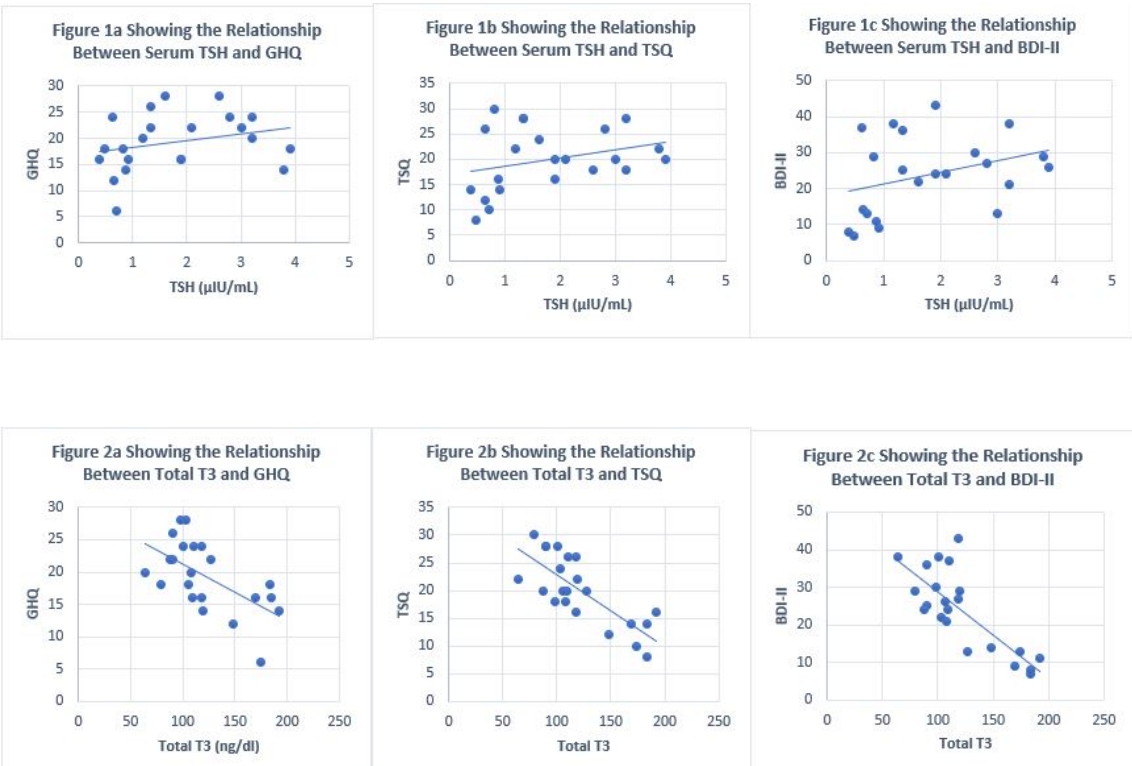

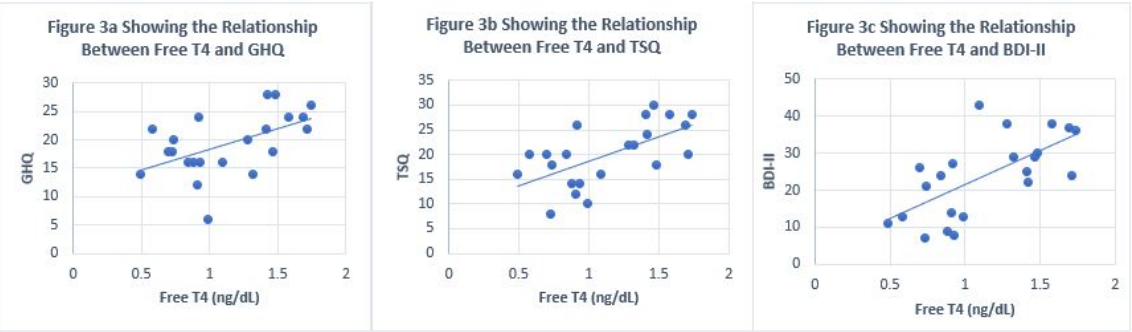